\title{
Sizing a Hybrid Renewable Energy System by a Coevolutionary Multiobjective Optimization Algorithm
}

\author{
Wenhua Li $\mathbb{D}^{1},{ }^{1}$ Guo Zhang, ${ }^{1}$ Xu Yang, ${ }^{1}$ Zhang Tao, ${ }^{1,2}$ and Hu Xu $\mathbb{D}^{3}$ \\ ${ }^{1}$ College of Systems Engineering, National University of Defense Technology, Changsha 410073, China \\ ${ }^{2}$ Hunan Key Laboratory of Multi-Energy System Intelligent Interconnection Technology, Changsha 410073, China \\ ${ }^{3}$ State Key Laboratory of Astronautic Dynamics, Xi'an 710043, China
}

Correspondence should be addressed to Wenhua Li; liwenhua1030@aliyun.com

Received 7 September 2020; Revised 15 September 2020; Accepted 23 January 2021; Published 10 February 2021

Academic Editor: Christos Volos

Copyright ( $\odot 2021$ Wenhua Li et al. This is an open access article distributed under the Creative Commons Attribution License, which permits unrestricted use, distribution, and reproduction in any medium, provided the original work is properly cited.

Hybrid renewable energy system (HRES) arises regularly in real life. By optimizing the capacity and running status of the microgrid (MG), HRES can decrease the running cost and improve the efficiency. Such an optimization problem is generally a constrained mixed-integer programming problem, which is usually solved by linear programming method. However, as more and more devices are added into MG, the mathematical model of HRES refers to nonlinear, in which the traditional method is incapable to solve. To address this issue, we first proposed the mathematical model of an HRES. Then, a coevolutionary multiobjective optimization algorithm, termed CMOEA-c, is proposed to handle the nonlinear part and the constraints. By considering the constraints and the objective values simultaneously, CMOEA-c can easily jump out of the local optimal solution and obtain satisfactory results. Experimental results show that, compared to other state-of-the-art methods, the proposed algorithm is competitive in solving HRES problems.

\section{Introduction}

Energy shortages and environmental pollution problems have become increasingly severe in recent years. Microgrid $(\mathrm{MG})$ technology [1] has received more and more attention to improving the rate of renewable energy usage, especially in large-scale wind and solar energy. MG is a small power supply and consumption system that contains a variety of components, e.g., electrical loads, energy storage devices, and renewable energy. So far, there have been many pieces of research on microgrid energy management. However, how to plan the capacity of the microgrid and consider the running operation of the microgrid in the design stage has become an urgent problem.

Hybrid renewable energy system (HRES) [2] aims to optimize the installed capacity of components and energy management of the MG system during the planning phase. The main problem is to determine a set of optimal configurations, including whether to install a component and the capacity to install [3]. The main problem in microgrid energy management is how to formulate a power generation and scheduling plan without affecting the stable operation of the system.

To address the above problem, many studies have proposed and improved the HRES model [4, 5]. Generally speaking, this problem is mixed-integer programming, so it can be optimized by traditional solving tools, e.g., branchand-cut [6]. However, with the update of microgrid components, the HRES model has increasingly shown nonlinear characteristics, which cannot be solved directly by traditional linear programming methods. It is reported that through the transformation, which transfers the nonlinear constraints and objective functions to their linear form, some simple nonlinear problems can be solved by traditional linear programming methods. However, such transformation is limited and loses the information of the original problems. On the other hand, the HRES problem is a typical multiobjective optimization problem [3]. Decision makers (DMs) need to consider the full life cycle cost, power reliability, pollution emission, and so on simultaneously [7]. 
The traditional linear programming method usually converts multiobjective optimization problems to singleobjective one by summing up objectives with a group of given weights [8]. That is, DM gives a set of weight vectors in advance, and then a scalar function is constructed for optimization. Although this method is simple, it has many drawbacks. Firstly, due to the inconsistency of various objective functions, it is difficult to determine the weights, resulting in poor robustness of the single-objective optimization problem. The use of larger weights will amplify the influence of the objective function noise, leading to unilateral preference. Secondly, it is often difficult for decision makers to give appropriate preference weights in advance [9].

Evolutionary multiobjective optimization (EMO) algorithm has gained more and more attention in dealing with real-world engineering problems with nonlinear objective functions and constraints [10]. Multiobjective optimization problems (MOPs) which require the simultaneous optimization of multiple objectives are common in real-world applications. Without loss of generality, a MOP can be formulated as follows:

$$
\begin{aligned}
\text { Minimize } \quad F(\mathbf{x}) & =\left\{f_{1}(\mathbf{x}), f_{2}(\mathbf{x}), \ldots, f_{m}(\mathbf{x})\right\}, \\
\text { s.t. } \quad \mathbf{x} & =\left(x_{1}, x_{2}, \ldots, x_{n}\right) \in \Omega,
\end{aligned}
$$

where $\Omega$ denotes the feasible decision space, $m$ is the number of objectives, and $\mathbf{x}$ is a decision vector consisting of $n$ decision variables $x_{i}$. A solution $\mathbf{x}_{\mathbf{a}}$ is said to Pareto dominate another solution $\mathbf{x}_{\mathbf{b}}$ if $\forall i=1,2, \ldots, m, f_{i}\left(\mathbf{x}_{\mathbf{a}}\right) \leq f_{i}\left(\mathbf{x}_{\mathbf{b}}\right)$ and $\exists j=1,2, \ldots, m, f_{j}\left(\mathbf{x}_{\mathbf{a}}\right)<f_{j}\left(\mathbf{x}_{\mathbf{b}}\right)$. The images of Pareto optimal solution set (PS) in the objective space are termed the Pareto optimal front (PF).

So far, a large number of works have been done to find a well-distributed PF of a MOP without constraints, which can be roughly categorized as (i) Pareto dominance-based algorithms, e.g., NSGA-II [11] and SPEA2 [12], (ii) indicator-based algorithms, e.g., IBEA [13], and (iii) decomposition-based methods, e.g., MOEA/D $[14,15]$. Although the proposed EMO algorithms can well solve the benchmark problems without constraints [16], they are not specially designed for MOPs with constraints. To deal with constraints by EMO algorithms, many approaches have been proposed. Among them, penalty function $[17,18]$ may be the most representative and easiest way, which introduces a penalty term into the objective function to penalize constraint violations on a minimization problem. The introduction of the penalty term enables us to transform a constrained optimization problem into an unconstrained one. However, it is very difficult to strike the right balance between objective and penalty functions. Other approaches like $\epsilon$-constraints [19] and stochastic ranking [20] can somehow deal with the constraints. Another effective way to deal with the constraints is to modify the dominance relationship [21], which has been proved effective and efficient. Specifically, there are two basic rules to calculate the dominance relationship. Solutions satisfying the constraints dominate unsatisfied solutions. If both solutions satisfy the constraints, then use the basic rule to compare their objective values.
In addition, it is still an open issue to solve mixed-integer programming problem through EMO [22, 23]. Compared to continuous problems, the decision spaces of mixed-integer problems are separated. As shown in Figure 1, a very simple example is given with only two integer variables. In this case, each feasible region contains only one solution. The number of feasible regions will exponentially increase as the number of decision variables grows. In general, we set the population size to 100 to optimize, which is largely insufficient for the whole decision space. Moreover, since feasible regions in the decision space are separated, it is difficult for EMO algorithms to continuously search the whole decision space.

In this paper, we first proposed the mathematical model of an HRES. We consider the building and running cost of this system as well as the energy supply stability. This HRES model is a nonlinear and mixed-integer multiobjective optimization problem. To solve this problem, we proposed a coevolutionary EMO algorithm with modified dominance relationship to deal with the constraints, termed CMOEA-c. Specifically, there is an assistant archive which aims to explore the whole decision space and help jump out of the local optimal solution. In addition, we compared the performance of the proposed algorithm with other state-of-theart algorithms. Experimental results show that our model and algorithm are effective and efficient.

The rest of this paper is structured as follows. A mathematical model of a hybrid renewable energy system is proposed and specifically explained in Section 2. In Section 3 , the proposed coevolutionary multiobjective optimization algorithm is illustrated, followed by Section 4, which shows the experiment setting and the result analysis. Finally, a conclusion of this work is given in Section 5.

\section{Mathematical Model of the Hybrid Renewable Energy System (HRES)}

A typical HRES model includes the following components, i.e., the external electricity grid, renewable power generation, user load, diesel generator (DE), and energy storage system (ESS), as shown in Figure 2. We need to first determine the installed capacities of these components. Then, we can optimize the control strategy of ESS and DE to minimize the running cost of this HRES.

2.1. Constraints. Due to the randomness and intermittent nature of renewable energy, the power supply is often unstable [24], and it is difficult to meet energy demand all the time with renewable energy alone. With the help of ESS, the gap between supply and demand can be eased to a certain extent. In order to improve the reliability of power supply, MG must be connected to an external power grid. In this process, to ensure safety, the maximum transmission capacity is limited within a certain range.

$$
X_{\text {sell }} P_{\text {grid }}^{\min } \leq P_{\text {grid }}(t) \leq X_{\text {sell }} P_{\text {grid }}^{\max }
$$

where $P_{\text {grid }}(t)$ is the exchanged power between external grid and $M G$. $P_{\text {grid }}^{\min }$ and $P_{\text {grid }}^{\max }$ represent the minimum and maximum power of the interaction respectively. $X_{\text {sell }}$ is a 


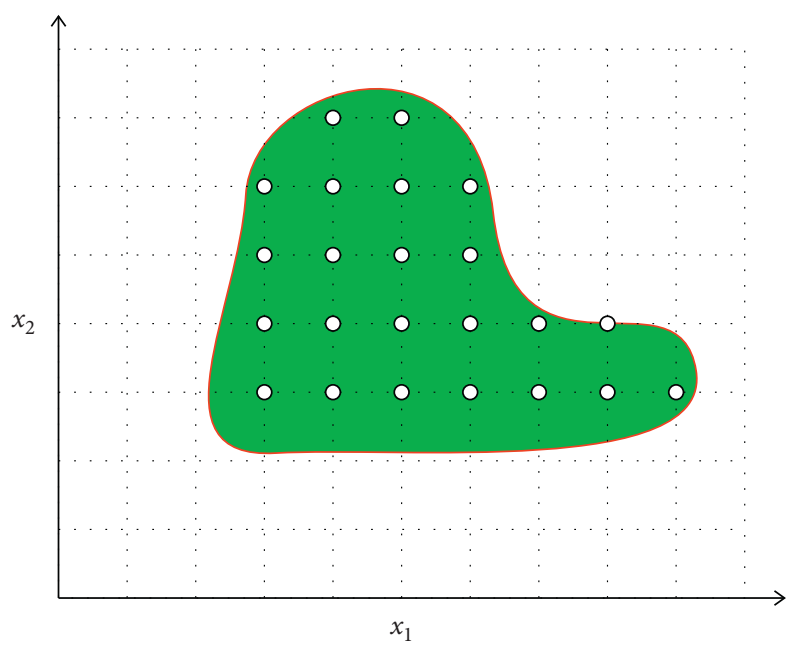

Figure 1: Decision space of a mixed-integer programming problem.

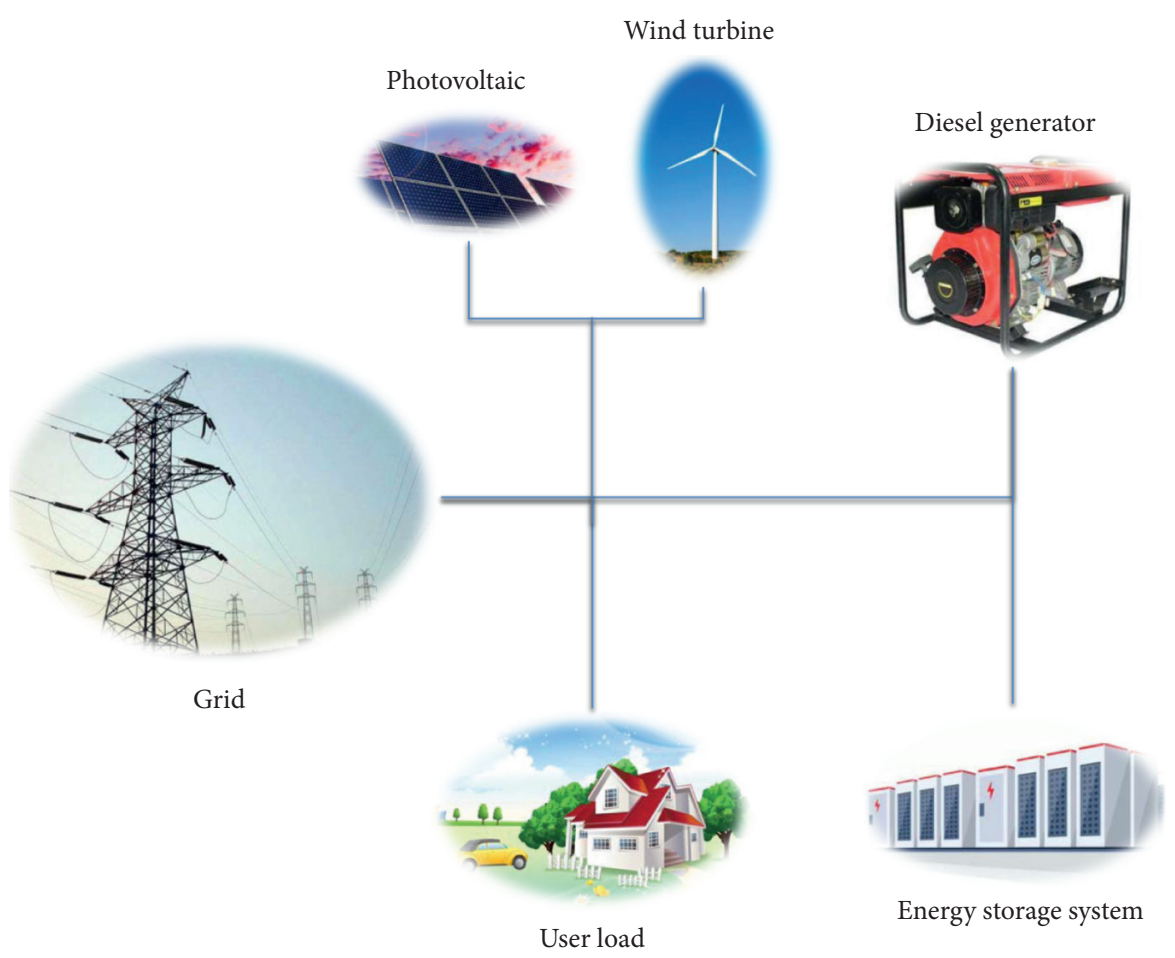

Figure 2: Physical structure of an HRES.

binary variable that indicates whether the MG can sell electricity to the grid. $P_{\text {grid }}^{\min }$ could be negative which means that the MG can sell electricity to the external grid.

Renewable energy includes photovoltaic and wind turbine; the main constraint is the minimum and maximum installed capacity.

$$
\begin{gathered}
A_{\min }^{\mathrm{pv}} \cdot X_{\mathrm{pv}} \leq A_{\mathrm{pv}} \leq A_{\mathrm{max}}^{\mathrm{pv}} \cdot X_{\mathrm{pv}}, \\
P_{\mathrm{pv}}(t) \leq A_{\mathrm{pv}} C p_{\mathrm{rat}}^{\mathrm{pv}},
\end{gathered}
$$

where $A_{\mathrm{pv}}$ is the installed area of photovoltaic; $X_{\mathrm{pv}}$ is a binary variable presenting if the photovoltaic equipment is installed or not; and $C p_{\mathrm{rat}}^{\mathrm{pv}}$ is the transfer efficiency of photovoltaic. The constraints of the wind turbine can be expressed as

$$
\begin{aligned}
N_{\min }^{\mathrm{wt}} \cdot X_{\mathrm{wt}} & \leq N_{\mathrm{wt}} \leq N_{\mathrm{max}}^{\mathrm{wt}} \cdot X_{\mathrm{wt}}, \\
P_{\mathrm{wt}}(t) & \leq C p_{\mathrm{rat}}^{\mathrm{wt}} \cdot N_{\mathrm{wt}},
\end{aligned}
$$

where $N_{\mathrm{wt}}$ and $C p_{\mathrm{rat}}^{\mathrm{wt}}$ represent the installed number and rated capacity of the wind turbine, respectively. $X_{\mathrm{wt}}$ is the selecting state of the wind turbine, where $X_{\mathrm{wt}}=0$ means that wind turbine is not installed in this HRES.

Similarly, the constraints of diesel generator are listed as follows: 


$$
\begin{gathered}
N_{\text {min }}^{\mathrm{de}} \cdot X_{\mathrm{de}} \leq N_{\mathrm{de}} \leq N_{\max }^{\mathrm{de}} \cdot X_{\mathrm{de}}, \\
P_{\mathrm{de}}(t) \leq C p_{\mathrm{rat}}^{\mathrm{de}} \cdot N_{\mathrm{de}} .
\end{gathered}
$$

Energy storage system (ESS) in an MG can not only store excess power but also supply power as a backup when the power generation is insufficient. With the help of the ESS, HRES can run more flexibly and stably. The main constraints are shown as follows:

$$
\begin{aligned}
P_{\mathrm{ess}}^{\mathrm{min}} \cdot X_{\mathrm{ess}} & \leq P_{\mathrm{ess}}(t) \leq P_{\mathrm{ess}}^{\mathrm{max}} \cdot X_{\mathrm{ess}}, \\
P_{\mathrm{ess}}^{\mathrm{max}} & =C p_{\mathrm{rat}}^{\mathrm{ess}} \cdot N_{\mathrm{ess}}, \\
E_{\mathrm{ess}}^{\mathrm{min}} \cdot X_{\mathrm{ess}} & \leq E_{\mathrm{ess}}(t) \leq E_{\mathrm{ess}}^{\mathrm{max}} \cdot X_{\mathrm{ess}}, \\
E_{\mathrm{ess}}(t+1) & =E_{\mathrm{ess}}(t)\left(1-\epsilon_{\mathrm{ess}}\right)+\eta_{\mathrm{ess}} P_{\mathrm{ess}}(t) \Delta t,
\end{aligned}
$$

where $P_{\text {ess }}^{\min }$ and $P_{\text {ess }}^{\max }$ are the minimum and maximum power of charging and discharging, respectively. $E_{\text {ess }}(t)$ means the energy storage capacity at $t$. In addition, $\epsilon_{\text {ess }}$ is the selfdischarge rate of ESS, which is provided by the manufacturer.

Parameter $\eta_{\text {bess }}$ denotes the charging and discharging efficiency of the ESS, which changes according to the state (charging and discharging) of the ESS.

$$
\eta_{\text {bess }}= \begin{cases}\eta_{\text {bess }}^{c}, & P_{\text {bess }}(t)>0 \\ \eta_{\text {bess }}^{d}, & P_{\text {bess }}(t) \leq 0\end{cases}
$$

where $\eta_{\text {bess }}^{c}$ and $\eta_{\text {bess }}^{d}$ denote the charge/discharge efficiency of the energy storage system ranging from 0 to 1 .

The power balance constraint refers to balancing the power supply and demand as shown in the following equation:

$$
P_{\mathrm{pv}}(t)+P_{\mathrm{wt}}(t)+P_{\mathrm{de}}(t)+P_{\text {grid }}(t)+P_{\text {lack }}(t)=P_{\text {load }}(t)+P_{\text {ess }}(t),
$$

where $P_{\mathrm{pv}}(t), P_{\mathrm{wt}}(t), P_{\mathrm{de}}(t), P_{\text {grid }}(t), P_{\text {load }}(t)$, and $P_{\text {ess }}(t)$ represent the power of photovoltaic, wind turbine, diesel generator, interaction with external grid, users' load, and the energy storage system, respectively.

2.2. Objective Functions. In this HRES model, we consider two objective functions, one is to minimize the life cycle cost, and the other is to minimize the rate of lack of electricity. Specifically, the life cycle cost includes the investment cost and maintenance cost, which is calculated as

$$
\begin{aligned}
& C_{\mathrm{INV}}=C_{\mathrm{INV}}^{\mathrm{pv}}+C_{\mathrm{INV}}^{\mathrm{wt}}+C_{\mathrm{INV}}^{\mathrm{ess}}+C_{\mathrm{INV}}^{\mathrm{de}}, \\
& C_{\mathrm{INV}}^{\mathrm{pv}}=\mathrm{CRF}^{\mathrm{pv}} \cdot C p_{\mathrm{rat}}^{\mathrm{pv}} \cdot A_{\mathrm{pv}} \cdot C_{\mathrm{pv}}, \\
& C_{\mathrm{INV}}^{\mathrm{wt}}=\mathrm{CRF}^{\mathrm{wt}} \cdot C p_{\mathrm{rat}}^{\mathrm{wt}} \cdot N_{\mathrm{wt}} \cdot C_{\mathrm{wt}}, \\
& C_{\mathrm{INV}}^{\mathrm{de}}=\mathrm{CRF}^{\mathrm{de}} \cdot C p_{\mathrm{rat}}^{\mathrm{de}} \cdot N_{\mathrm{de}} \cdot C_{\mathrm{de}}, \\
& C_{\mathrm{INV}}^{\mathrm{ess}}=\mathrm{CRF}^{\mathrm{ess}} \cdot C p_{\mathrm{rat}}^{\mathrm{ess}} \cdot N_{\mathrm{ess}} \cdot C_{\mathrm{ess}},
\end{aligned}
$$

where $C_{\mathrm{INV}}$ and $C_{\text {equipment }}$ are the total and unit installed costs of each component. In this study, we only consider two-day energy management. Thus, CRF is introduced to calculate the annual cost, which is given by decision makers.
The maintenance cost of HRES can be expressed as

$$
\begin{aligned}
& C_{\mathrm{MTN}}=C_{\mathrm{MTN}}^{\mathrm{pv}}+C_{\mathrm{MTN}}^{\mathrm{wt}}+C_{\mathrm{MTN}}^{\mathrm{ess}}+C_{\mathrm{MTN}}^{\mathrm{de}}+C_{\mathrm{op}}^{\mathrm{grid}}, \\
& C_{\mathrm{MTN}}^{\mathrm{pv}}=\mathrm{CRF}^{\mathrm{pv}} \cdot P_{\mathrm{pv}} \cdot C_{\mathrm{mtn}}^{\mathrm{pv}}, \\
& C_{\mathrm{MTN}}^{\mathrm{wt}}=\mathrm{CRF}^{\mathrm{wt}} \cdot P_{\mathrm{wt}} \cdot C_{\mathrm{mtn}}^{\mathrm{wt}}, \\
& C_{\mathrm{MTN}}^{\mathrm{de}}=\mathrm{CRF}^{\mathrm{de}} \cdot P_{\mathrm{de}} \cdot C_{\mathrm{mtn}}^{\mathrm{de}}, \\
& C_{\mathrm{MTN}}^{\mathrm{ess}}=\mathrm{CRF}^{\mathrm{ess}} \cdot P_{\mathrm{ess}} \cdot C_{\mathrm{mtn}}^{\mathrm{ess}}, \\
& C_{\mathrm{op}}^{\mathrm{Grid}}=C_{\mathrm{Pur}}^{\mathrm{grid}} \cdot P_{\mathrm{Pur}}^{\mathrm{grid}}-C_{\mathrm{Sal}}^{\mathrm{grid}} \cdot P_{\mathrm{Sal}}^{\mathrm{grid}},
\end{aligned}
$$

where $C_{\text {op }}^{\text {Grid }}$ is the cost of purchasing and selling electricity with the grid.

Another objective is to minimize the rate of lack of electricity. Therefore, the objective functions of HRES are written as follows:

$$
\begin{aligned}
& \operatorname{Min} f_{1}=C_{\mathrm{INV}}+C_{\mathrm{MTN}}, \\
& \operatorname{Min} f_{2}=\sum P_{\text {lack }}(t) .
\end{aligned}
$$

Generally speaking, these two objectives are conflicting, which means we cannot simply optimize one objective. Reasonably, the higher the cost of investment and maintenance, the lower the lack of electricity.

\section{The Constrained Coevolutionary Multiobjective Optimization Algorithm}

3.1. Motivation and Framework. There are many approaches to deal with constraints in a MOP as we have reviewed in Section 1. These approaches can obtain favorable results on benchmark problems. However, real-world engineering problems are generally complex and multimodal. Generally, the feasible regions of these problems are usually separated from each other in objective space and decision space. Figure 3 explains the objective space of a two-objective realworld engineering problem, where the red line means the Pareto front. As shown in Figure 3, there are two feasible regions $B$ and $D$ in the objective space, separated by infeasible region $C$. For traditional EMO algorithms with constraint-handling strategies, it is really hard for populations to enter into region $B$ due to the existence of infeasible region $C$. Generally speaking, at the beginning of the algorithm run, most of the solutions will randomly locate outside region $C$. As the algorithms adopt the constrainthandling methods, solutions will converge to the bound of region $C$ and $D$ and keep exploring region $D$. As a result, the algorithm is stuck into the local optimal solution and it is extremely hard to jump out.

To address this issue, we proposed a constrained coevolutionary multiobjective optimization algorithm, termed CMOEA-c, which introduces an archive to help explore the whole decision space. The thoughts of coevolution can be widely found in some state-of-the-art EMO algorithms [25-27], which is proved effective and efficient. Specifically, we do not process the constraints in this archive. This archive aims to find the Pareto optimal front of a normal MOP without considering the constraints. Therefore, solutions in the archive 


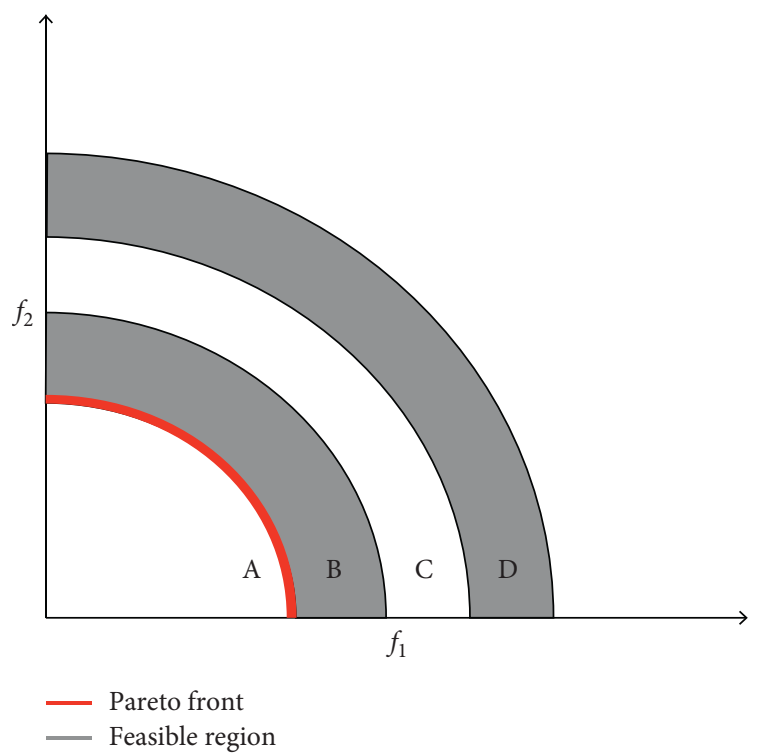

Figure 3: Illustration of a constraint multiobjective problem, where the feasible regions are separate.

will quickly converge to the true Pareto front, namely, the red line in Figure 3. In the beginning of the evolution process, solutions in the main population and the archive are randomly generated and may locate on regions $C$ and $D$. As the algorithm runs, solutions in main population will converge to the optimal solution of region $D$, which is similar to that of traditional constrained EMO. As a comparison, since solutions in the archive do not consider the constraints, they will directly go through region $C$. Once the solutions in the archive enter into region $B$, this information will pass to the main population through the sharing function. As a result, the main population will quickly move to region $B$ and then converge to the true Pareto optimal front. The framework of the proposed algorithm is explained in Algorithm 1.

As we can see from Algorithm 1, the main population and the introduced archive will coevolve by sharing their offspring (line 8 -9). Therefore, solutions in region $\mathrm{B}$ can be added to the main population and help to converge to the true Pareto front. We will explain the selection strategy in the following section in detail.

3.2. Environmental Selection. After combining with the offspring, we need to update the population and the assistant archive by environmental selection, namely, maintain the population size to a certain number, shown in Algorithm 2. There is one major difference between the update process of the population and the archive. Specifically, there are two steps. Firstly, we need to calculate the dominance ranking information and crowding distance of the population. Secondly, if the number of nondominated solutions exceeds $N$, then we truncate it to $N$ according to crowding distance; otherwise, we will firstly choose all nondominated solutions and then select other solutions by crowding distance to make sure the population size is equal to $N$.

Notably, for the main population, we use modified dominance relationship. That is, a feasible solution dominates an infeasible solution; if two solutions are feasible, then we use objective values to calculate the dominance relationship. As for the assistant archive, we do not consider whether the solution is feasible or not. The calculation of the dominance relationship is the same as the traditional ones.

3.3. Solving HRES by CMOEA-c. The main problem to solve HRES problem by EMO algorithms is to encode a solution and design the mutation strategies. Since the HRES model in the work is complex, we use a structure to represent the solution, which can be seen in Figure 4.

As we can see from Figure 4, there are five parts describing a solution for an HRES. The first two parts indicate the installed information of an HRES, including whether to install the equipment or not and the installed capacity. Specifically, 0 means that the HRES does not select to install this equipment, and vice versa. Besides, the running state of this HRES is determined by the first four parts, where ESS running state and DE running state participate. Notably, some instrumental variables can help to calculate the objective function values but do not take part in the mutation, which are stored in the final part of the solution, e.g., wind power output, photovoltaic power output, and electricity prices. Specifically, the values of these variables change when the equipment select state and installed capacity change.

As we can see from Figure 4, there are several variable types in a solution for an HRES. Thus, simply applying the same mutation strategy to evolve the solution is low-efficient and unreasonable. To accelerate the searching process, we divide them into several parts and perform different mutation strategies. Specifically, for the equipment select states, we use a small mutation probability $p_{m}=0.1$ because the change of equipment select state will significantly affect the running state of an HRES. In addition, for mixed-integer variables, we will perform integrity checking after the mutation to ensure the solution is feasible. The mutation probabilities for mixed-integer variables and continuous variables are 0.5 and 1 , respectively.

\section{Experiments}

4.1. Parameter Setting. To examine the performance of CMOEA-c, we choose SRA [20] and SPEA2+ ${ }_{\text {pf }}$ (SPEA2+ [28] with penalty function) as the competitor algorithms. Specifically, SRA is the most representative algorithm using stochastic ranking to deal with the constraints; SPEA2+ is chosen as the latest MOEA where the penalty function is added to each objective directly. All parameters are set to default values according to the original paper for fairness. For all algorithms, population size is 100 and the number of function evaluation is 20000. Each experiment is run for 31 times and the mean value is adopted for comparison and presentation. The parameters of this HRES are listed in Table 1. Besides, the generation power of the wind turbine and photovoltaic is related to local weather, which is shown in Figure 5. Moreover, the purchasing price and selling price of electricity are given as well. 


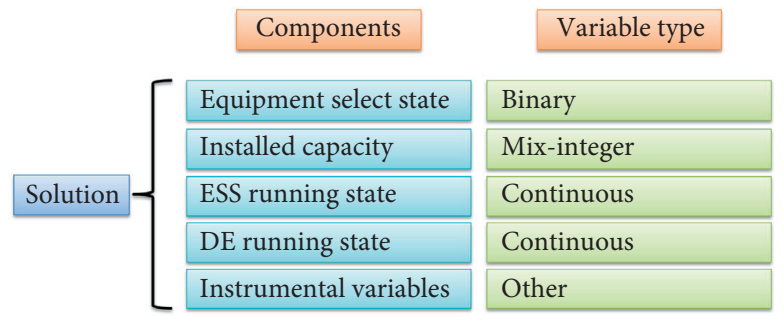

FIgURE 4: Illustration of solution encoding method and the variable type.

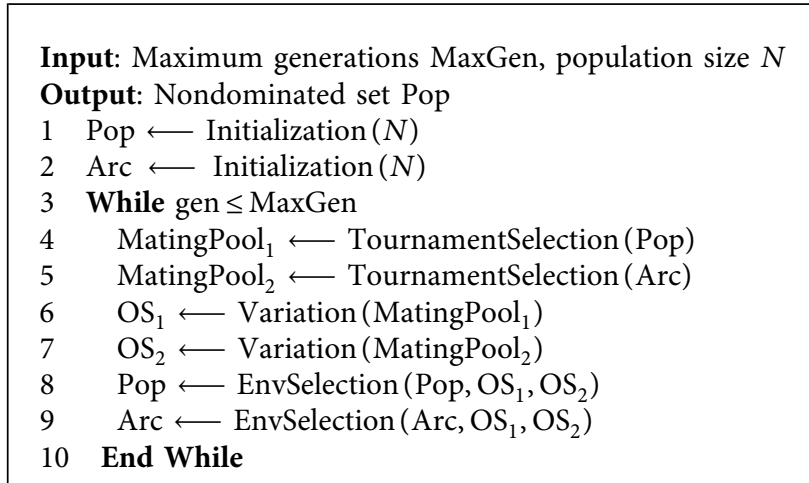

Algorithm 1: General framework.

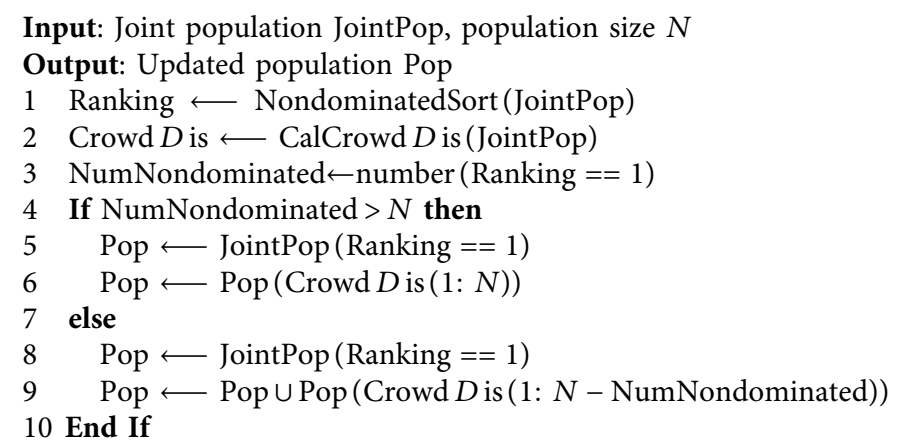

Algorithm 2: Environmental selection.

4.2. Performance of the Proposed Algorithm. To examine the performance of CMOEA-c, we choose two constraint EMO algorithms as the competitors. It is worth mentioning that all experiments are executed 31 times independently. We choose the mean result among 31 times to present, which is shown in Figure 6.

As we can see from Figure 6, the Pareto front obtained by CMOEA-c is better than that of SRA. Moreover, the result obtained by SPEA $2+_{\mathrm{pf}}$ is far away from the true Pareto front. This is because directly adding penalty function to objective functions cannot provide effective information to lead the search. As a result, there is a large probability for SPEA $2+_{\mathrm{pf}}$ to get into the local optimal solution in the early stage of the algorithm run.

Compared to SRA, CMOEA-c got better results. This is because the introduction of the assistant archive can help to explore the whole decision space. As we mentioned in Section 3, since the feasible regions are generally separated, once the constraint population converges to the optimal solution, the archive will keep search without considering the constraints. However, there is no such mechanism in SRA to help jump from one feasible region to another. Therefore, CMOEA-c can obtain better results.

4.3. Analysis of the Obtained Result. To further analyse the result obtained by CMOEA-c, we use Technique for Order of Preference by Similarity to Ideal Solution (TOPSIS) [29] to pick the ideal solution as the final optimal result. Other approaches like knee point $[30,31]$ are also suitable for selecting satisfactory solutions. Specifically, the basic principle of TOPSIS is to sort solutions by calculating the 
TABLE 1: The parameter values of all equipment used in HRES.

\begin{tabular}{|c|c|c|c|c|c|}
\hline Equipment & Unit cost investment & Unit cost maintenance & Min capacity & Max capacity & Lifetime \\
\hline Wind turbine & 55 & 5 & 0 & 100 & 5 \\
\hline Photovoltaic & 14 & 0.1 & 0 & $10000\left(\mathrm{~m}^{2}\right)$ & 25 \\
\hline Energy storage system & 3 & 0.1 & 0 & 100 & 9 \\
\hline Diesel generator & 2000 & 0.05 & 0 & 50 & 20 \\
\hline Power grid & - & - & - & $10000(\mathrm{~kW})$ & - \\
\hline
\end{tabular}

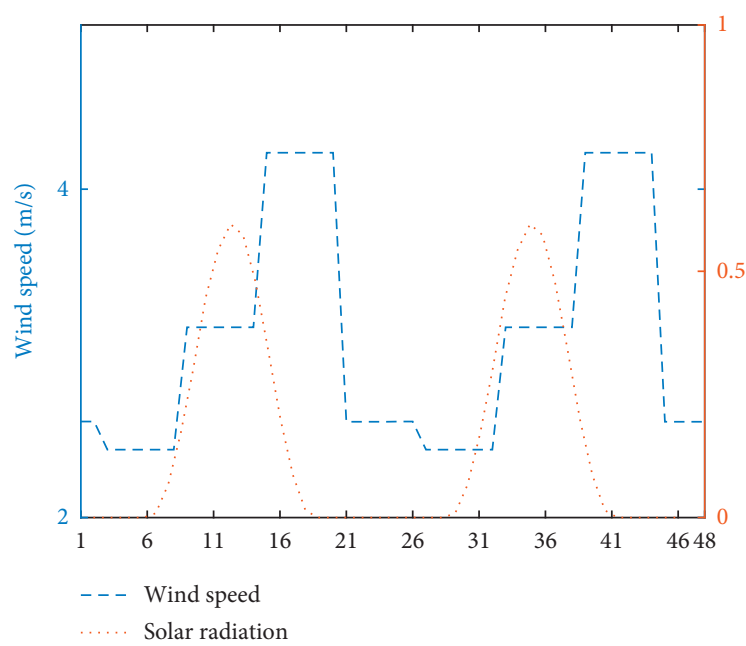

(a)

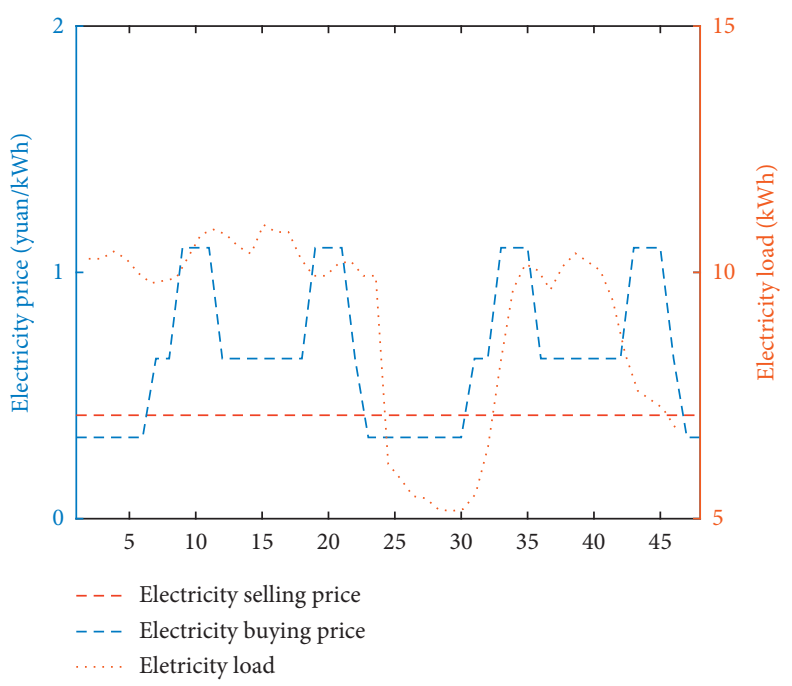

(b)

FIGURE 5: Running data of HRES in this study. (a) Wind speed and solar radiation. (b) User load and electricity price.

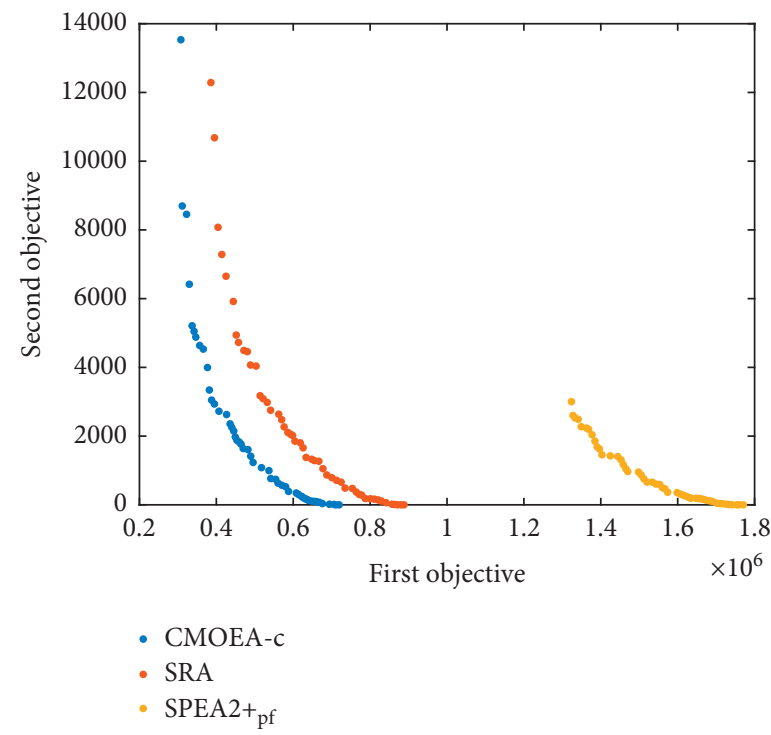

Figure 6: Pareto fronts obtained by CMOEA-c, SRA, and SPEA2+pf.

distances between the evaluating solution and the optimal and the worst solutions. If the evaluating solution is closest to the optimal solution and farthest from the worst solution, it is the best; otherwise, it is not an optimal solution. The running status of all components is presented in Figure 7.

As we can see from Figure 7(b), when the HRES is connected with the grid, it is more likely to sell electricity to the grid when the electricity purchasing price is high. This is because the system can minimize the running cost in this way. It is worth mentioning that wind turbine power generation is very close to electricity, and thus other components of the HRES are running with a low maintenance cost. In addition, when the HRES is isolated from the main grid, it needs to balance the power supply and demand all the time. 


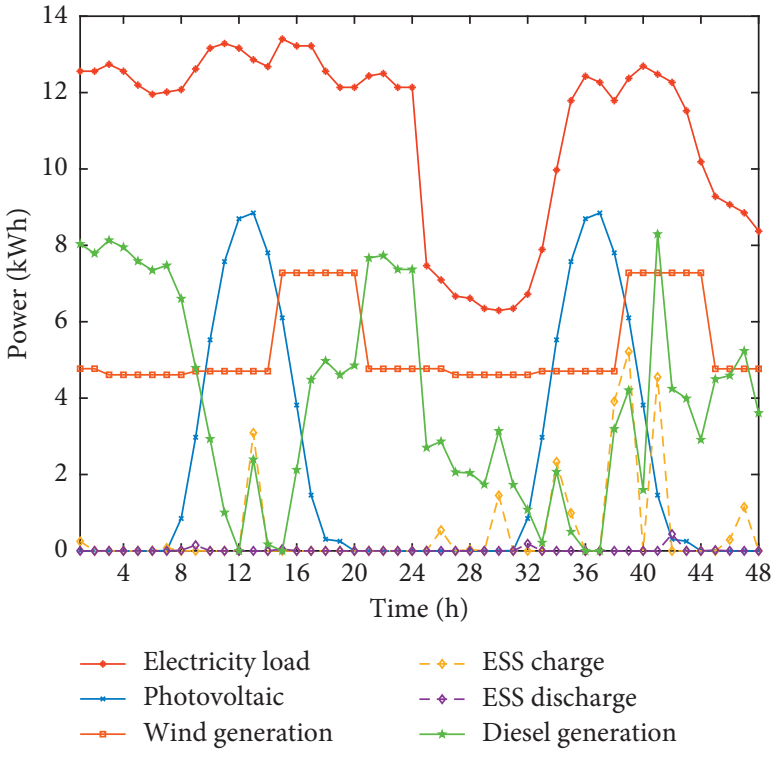

(a)

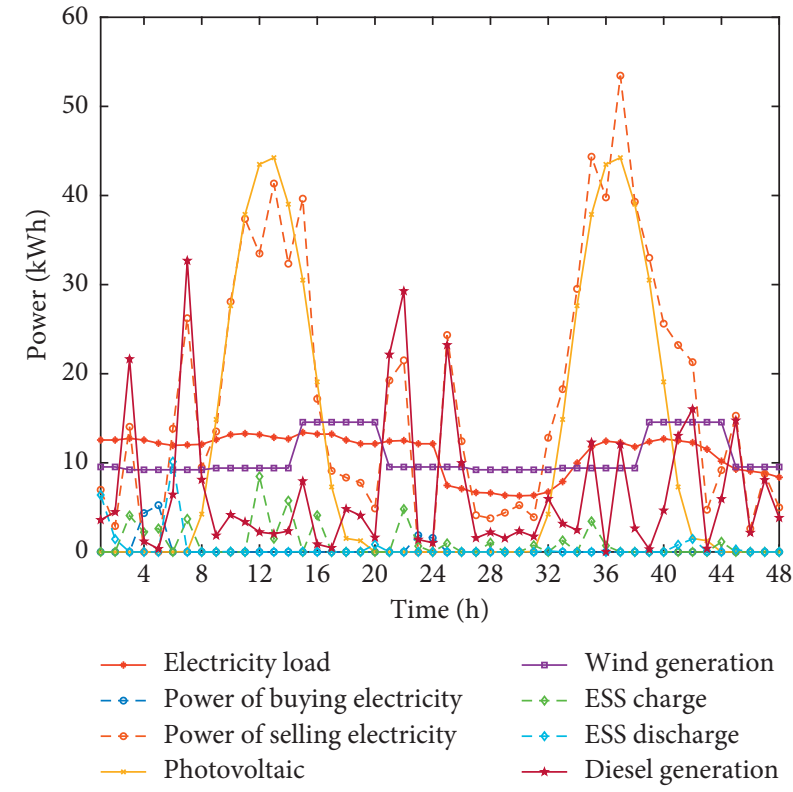

(b)

FIGURE 7: The equipment running status of HRES in this study. (a) Isolated microgrid. (b) Grid-connected microgrid.

To minimize the running cost of diesel generator and energy storage system, renewable energy generation (wind power and photovoltaic) is matched with the electricity load as much as possible as we can see from Figure 7(a). In conclusion, the proposed HRES model is effective which can be used to optimize the configuration in the designing stage. Moreover, our proposed CMOEA-c can effectively and efficiently solve HRES problems with nonlinear parts.

\section{Conclusion}

Microgrid is an effective tool to make better use of renewable energy. However, it is a tough task to determine the installed capacity of components, e.g., the number of wind turbine and the area of the photovoltaic cell. Thus, a hybrid renewable energy system (HRES) is proposed, which is generally nonlinear and large-scale. In addition, as more and more aspects are taken into consideration, an HRES problem needs to optimize more than one objective simultaneously. Therefore, traditional methods like linear programming are not suitable to solve this kind of problems.

In this paper, we first proposed the mathematical model of a hybrid renewable energy system, which has nonlinear parts. We consider two objectives to optimize, one is to minimize building and daily running cost, and another is to minimize the rate of lack of electricity. To solve this problem, we also proposed a coevolutionary multiobjective algorithm. Specifically, we use an archive to help explore the whole decision space. To examine the effectiveness of our proposed algorithm, several experiments are conducted, which show that the proposed algorithm can effectively find the Pareto optimal front of HRES problems.

We only consider a two-day (48 hours) energy management problem for HRES in this study. However, a longer period is necessary, e.g., one year. Such problems are large-scale which have more than 10000 decision variables. So far, it is really difficult to solve this issue with evolutionary algorithms $[32,33]$, which is our future work. On the other hand, the decision variables of the HRES problem studied in this work are highly correlated. Specifically, the selecting state can significantly affect other decision variables. For such a mixedinteger problem, we need to develop a more effective algorithm.

\section{Data Availability}

The research data used to support the findings of this study are included within the article.

\section{Conflicts of Interest}

The authors declare that they have no conflicts of interest.

\section{Acknowledgments}

This study was supported by the National Natural Science Foundation of China (61773390), the Young Talents of Hunan (2018RS3081), and the Scientific Research Project of National University of Defense Technology (ZZKY-ZX-11-04 and ZK18-02-09).

\section{References}

[1] E. Mengelkamp, J. Gärttner, K. Rock, S. Kessler, L. Orsini, and C. Weinhardt, "Designing microgrid energy markets: a case study: the brooklyn microgrid," Applied Energy, vol. 210, pp. 870-880, 2018.

[2] V. Khare, S. Nema, and P. Baredar, "Solar-wind hybrid renewable energy system: a review," Renewable and Sustainable Energy Reviews, vol. 58, pp. 23-33, 2016. 
[3] M. Ming, R. Wang, Y. Zha, and T. Zhang, "Multi-objective optimization of hybrid renewable energy system using an enhanced multi-objective evolutionary algorithm," Energies, vol. 10, no. 5, p. 674, 2017.

[4] R. Hosseinalizadeh, H. Shakouri G, M. S. Amalnick, and P. Taghipour, "Economic sizing of a hybrid (PV-WT-FC) renewable energy system (HRES) for stand-alone usages by an optimization-simulation model: case study of Iran," Renewable and Sustainable Energy Reviews, vol. 54, pp. 139-150, 2016.

[5] M. Amer, A. Namaane, and N. K. M'Sirdi, "Optimization of hybrid renewable energy systems (HRES) using PSO for cost reduction," Energy Procedia, vol. 42, pp. 318-327, 2013.

[6] S. Vigerske and A. Gleixner, "SCIP: global optimization of mixed-integer nonlinear programs in a branch-and-cut framework," Optimization Methods and Software, vol. 33, no. 3, pp. 563-593, 2018.

[7] R. Wang, G. Li, M. Ming, G. Wu, and L. Wang, "An efficient multi-objective model and algorithm for sizing a stand-alone hybrid renewable energy system," Energy, vol. 141, pp. 2288-2299, 2017.

[8] W. Li, T. Zhang, and R. Wang, "Energy management model of charging station micro-grid considering random arrival of electric vehicles," in Proceedings of the 2018 IEEE International Conference on Energy Internet (ICEI), pp. 29-34, Beijing, China, May 2018.

[9] W. Li, "Knee point-guided multiobjective optimization algorithm for microgrid dynamic energy management," Complexity, vol. 2020, Article ID 8877008, 2020.

[10] C. C. Coello, "Evolutionary multi-objective optimization: a historical view of the field," IEEE Computational Intelligence Magazine, vol. 1, no. 1, pp. 28-36, 2006.

[11] K. Deb, A. Pratap, S. Agarwal, and T. Meyarivan, "A fast and elitist multiobjective genetic algorithm: NSGA-II," IEEE Transactions on Evolutionary Computation, vol. 6, no. 2, pp. 182-197, 2002.

[12] E. Zitzler, M. Laumanns, and L. Thiele, "SPEA2: improving the strength pareto evolutionary algorithm," TIK-report, vol. 103, 2001.

[13] E. Zitzler and S. Künzli, "Indicator-based selection in multiobjective search," in Proceedings of the International Conference on Parallel Problem Solving from Nature, pp. 832-842, Birmingham, UK, September 2004.

[14] Q. Zhang and H. Li, "MOEA/D: a multiobjective evolutionary algorithm based on decomposition," IEEE Transactions on Evolutionary Computation, vol. 11, no. 6, pp. 712-731, 2007.

[15] R. Wang, "Localized weighted sum method for many-objective optimization," IEEE Transactions on Evolutionary Computation, vol. 22, no. 1, pp. 3-18, 2016.

[16] K. Li, R. Wang, T. Zhang, and H. Ishibuchi, "Evolutionary many-objective optimization: a comparative study of the state-of-the-art," IEEE Access, vol. 6, pp. 26194-26214, 2018.

[17] Q. Zhang, G. Pang, and G. Wang, "A novel sequential threeway decisions model based on penalty function," KnowledgeBased Systems, vol. 192, Article ID 105350, 2020.

[18] A. Panda and S. Pani, "A symbiotic organisms search algorithm with adaptive penalty function to solve multi-objective constrained optimization problems," Applied Soft Computing, vol. 46, pp. 344-360, 2016.

[19] W. Li, R. Wang, T. Zhang, M. Ming, and H. Lei, "Multiscenario microgrid optimization using an evolutionary multiobjective algorithm," Swarm and Evolutionary Computation, vol. 50, Article ID 100570, 2019.
[20] B. Li, K. Tang, J. Li, and X. Yao, "Stochastic ranking algorithm for many-objective optimization based on multiple indicators," IEEE Transactions on Evolutionary Computation, vol. 20, no. 6, pp. 924-938, 2016.

[21] Y. Tian, T. Zhang, J. Xiao, X. Zhang, and Y. Jin, “A coevolutionary framework for constrained multi-objective optimization problems," IEEE Transactions on Evolutionary Computation, vol. 25, no. 1, pp. 102-116, 2021.

[22] A. Salhi, G. Alsoufi, and X. Yang, "An evolutionary approach to a combined mixed integer programming model of seaside operations as arise in container ports," Annals of Operations Research, vol. 272, no. 1-2, pp. 69-98, 2019.

[23] K. L. Sadowski, M. C. van der Meer, N. H. Luong et al., "Exploring trade-offs between target coverage, healthy tissue sparing, and the placement of catheters in hdr brachytherapy for prostate cancer using a novel multi-objective model-based mixed-integer evolutionary algorithm," in Proceedings of the Genetic and Evolutionary Computation Conference, pp. 1224-1231, Berlin, Germany, July 2017.

[24] H.-Z. Wang, G.-Q. Li, G.-B. Wang, J.-C. Peng, H. Jiang, and Y.-T. Liu, "Deep learning based ensemble approach for probabilistic wind power forecasting," Applied Energy, vol. 188, pp. 56-70, 2017.

[25] R. Wang, W. Ma, M. Tan et al., "Preference-inspired coevolutionary algorithm with active diversity strategy for multi-objective multi-modal optimization," Information Sciences, vol. 546, pp. 1148-1165.

[26] Y. Liu, G. G. Yen, and D. Gong, "A multimodal multiobjective evolutionary algorithm using two-archive and recombination strategies," IEEE Transactions on Evolutionary Computation, vol. 23, no. 4, pp. 660-674, 2018.

[27] R. Wang, R. C. Purshouse, and P. J. Fleming, "Preferenceinspired coevolutionary algorithms for many-objective optimization," IEEE Transactions on Evolutionary Computation, vol. 17, no. 4, pp. 474-494, 2012.

[28] M. Kim, T. Hiroyasu, M. Miki, and S. Watanabe, "SPEA2+: Improving the performance of the strength pareto evolutionary algorithm 2," in International Conference on Parallel Problem Solving from Nature, pp. 742-751, Birmingham, UK, September 2004.

[29] D. Niu, Y. Li, S. Dai et al., "Sustainability evaluation of power grid construction projects using improved topsis and least square support vector machine with modified fly optimization algorithm," Sustainability, vol. 10, no. 1, p. 231, 2018.

[30] W. Li, R. Wang, T. Zhang, M. Ming, and K. Li, "Reinvestigation of evolutionary many-objective optimization: focus on the pareto knee front," Information Sciences, vol. 522, pp. 193-213, 2020.

[31] W. Li, T. Zhang, R. Wang, B. Wang, Y. Song, and X. Li, “A knee-point driven multi-objective evolutionary algorithm for flexible job shop scheduling," in Proceedings of the 2019 IEEE Symposium Series on Computational Intelligence (SSCI), pp. 1716-1722, Xiamen, China, December 2019.

[32] X. Zhang, Y. Tian, R. Cheng, and Y. Jin, "A decision variable clustering-based evolutionary algorithm for large-scale manyobjective optimization," IEEE Transactions on Evolutionary Computation, vol. 22, no. 1, pp. 97-112, 2016.

[33] R. Cheng, Y. Jin, and M. Olhofer, "Test problems for largescale multiobjective and many-objective optimization," IEEE Transactions on Cybernetics, vol. 47, no. 12, pp. 4108-4121, 2016. 